\title{
Revisiting a light NMSSM pseudoscalar at the LHC
}

\author{
Nils-Erik Bomark, ${ }^{a}$ Stefano Moretti, ${ }^{b}$ Shoaib Munir ${ }^{* c}$ and Leszek Roszkowski ${ }^{a}$ \\ ${ }^{a}$ National Centre for Nuclear Research \\ Hoża 69, 00-681 Warsaw, Poland \\ ${ }^{b}$ School of Physics \& Astronomy, University of Southampton \\ Southampton SO17 1BJ, UK \\ ${ }^{c}$ Department of Physics and Astronomy, Uppsala University \\ Box 516, SE-751 20 Uppsala, Sweden \\ E-mail: nbomark@fuw.edu.pl, s.moretti@soton.ac.uk, \\ shoaib.munir@physics.uu.se, leszek.roszkowski@fuw.edu.pl
}

\begin{abstract}
The discovery of a light, singlet-like pseudoscalar Higgs boson, $A_{1}$, of the Next-to-Minimal Supersymmetric Standard Model (NMSSM) could provide a hallmark signature of non-minimal supersymmetry. We review here the potential of the LHC to probe such a light $A_{1}$ in the decays of one of the heavier scalar Higgs bosons of the NMSSM. We find the production of pairs of the $A_{1}$, with a mass below $60 \mathrm{GeV}$ or so, via decays of the two lightest scalar states to be especially promising, for an integrated luminosity as low as 30/fb. For heavier masses, the decay of the heaviest scalar into a $Z$ boson and an $A_{1}$ could lead to its detection at the LHC.
\end{abstract}

Prospects for Charged Higgs Discovery at Colliders - CHARGED 2014,

16-18 September 2014

Uppsala University, Sweden

${ }^{*}$ Speaker. 


\section{Introduction}

The NMSSM contains an extra singlet Higgs superfield in addition to the two doublet superfields of the Minimal Supersymmetric Standard Model. As a result, there are a total of five neutral Higgs mass eigenstates: scalars $H_{i}$, with $i=1,2,3$, and pseudoscalars $A_{1,2}$, and a charged pair $H^{ \pm}$, in the model. The masses of the two new singlet-like states are generally very weakly constrained by the Higgs boson data from the Large Electron Positron collider or the Large Hadron Collider (LHC), and can be as low as a few GeV. We assess the scope of the detectability of a light, $\lesssim 150 \mathrm{GeV}, A_{1}$ of the NMSSM at the run 2 of the LHC with $\sqrt{s}=14 \mathrm{TeV}$. Through dedicated scans of the parameter space of the contrained NMSSM with non-universal Higgs masses (CNMSSM-NUHM), we found a considerable number of points containing such $A_{1}$ while also satisfying important experimental constraints. We then performed a detailed signal-to-background analysis for each of the main production and decay channels of $A_{1}$. Most notably, we employed the jet substructure method for detecting the $b$-quarks originating from $A_{1}$ decays, which considerably improves the experimental sensitvitiy.

\section{2. $A_{1}$ production channels in the model studied}

The soft supersymmetry (SUSY)-breaking Higgs potential of the NMSSM is written as

$$
V_{\text {soft }}=m_{H_{u}}^{2}\left|H_{u}\right|^{2}+m_{H_{d}}^{2}\left|H_{d}\right|^{2}+m_{S}^{2}|S|^{2}+\left(\lambda A_{\lambda} S H_{u} H_{d}+\frac{1}{3} \kappa A_{\kappa} S^{3}+\text { h.c. }\right),
$$

where $\lambda$ and $\kappa$ are dimensionless couplings and $A_{\lambda}$ and $A_{\kappa}$ are trilinear soft parameters. In the CNMSSM-NUHM the soft masses of the Higgs fields $m_{H_{u}}, m_{H_{d}}$ and $m_{S}$ are separated from the unified scalar mass parameter $m_{0}$ at the grand unification (GUT) scale. These three masses can be traded at the electroweak (EW) scale for the parameters $\tan \beta\left(\equiv v_{u} / v_{d}\right.$, with $v_{u}$ being the vacuum expectation value (VEV) of the $u$-type Higgs doublet and $v_{d}$ that of the $d$-type one), $\mu_{\text {eff }}$ ( $\equiv \lambda s$, with $s$ being the VEV of the singlet field) and $\kappa$. Similarly $A_{\lambda}{ }^{*}$ and $A_{\kappa}{ }^{*}$ (with the ${ }^{*}$ implying that these are defined at the GUT scale) are also disunified from the trilinear coupling parameter $A_{0}$. The CNMSSM-NUHM thus contains a total of nine continuous input parameters, which are given in table 1 along with their ranges scanned for this study. These ranges correspond to the "naturalness limit' of the model, where $\mathrm{H}_{2}$ with a mass consistent with that of the Higgs boson discovered at the LHC $[2,3]$ can be obtained without requiring large radiative corrections from the stop sector.

The tree-level mass-squared of the $A_{1}$ in the NMSSM is written in terms of the above parameters (defined at the SUSY-breaking scale), assuming negligible singlet-doublet mixing, as

$$
m_{A_{1}}^{2} \simeq \frac{A_{\lambda}}{2 s} v^{2} \lambda \sin 2 \beta+\kappa\left(2 v^{2} \lambda \sin 2 \beta-3 s A_{\kappa}\right),
$$

where $v \equiv \sqrt{v_{u}^{2}+v_{d}^{2}} \simeq 174 \mathrm{GeV}$. At the LHC, the $A_{1}$ can either be produced directly, preferably in the $g g \rightarrow b b A_{1}$ channel, owing to the possibility of a considerably enhanced $b \bar{b} A_{1}$ coupling [1] compared to the $g g A_{1}$ effective coupling. Alternatively, each of $H_{i}$, produced in the gluon-fusion (GF) mode, can also decay into $A_{1} A_{1}$ or $A_{1} Z$ pairs, when kinematically allowed. Here we will consider only these indirect production modes. 


\begin{tabular}{|c|c|c|c|c|}
\hline Parameter & $m_{0}(\mathrm{GeV})$ & $m_{1 / 2}(\mathrm{GeV})$ & $A_{0}(\mathrm{GeV})$ & $\mu_{\text {eff }}(\mathrm{GeV})$ \\
\hline Range & $200-2000$ & $100-1000$ & $-3000-0$ & $100-200$ \\
\hline \hline $\tan \beta$ & $\lambda$ & $\kappa$ & $A_{\lambda}{ }^{*}(\mathrm{GeV})$ & $A_{\kappa}{ }^{*}(\mathrm{GeV})$ \\
\hline $1-6$ & $0.4-0.7$ & $0.01-0.7$ & $-500-500$ & $-500-500$ \\
\hline
\end{tabular}

Table 1: The CNMSSM-NUHM input parameters and their scanned ranges.

In particular, in case of the decaying SM-like $H_{2}$, the mass measurement of $\sim 125 \mathrm{GeV}$ serves as an important kinematical handle. Removing this condition (for $H_{1}$ and $H_{3}$ ) reduces the sensitivity by a factor of 2 to 3 . The $A_{1} A_{1}$ pair thus produced decays via the $b \bar{b} b \bar{b}(4 b), b \bar{b} \tau^{+} \tau^{-}(2 b 2 \tau)$ and $\tau^{+} \tau^{-} \tau^{+} \tau^{-}(4 \tau)$ final state combinations. In the case of $A_{1} Z$ production, we only take the $Z \rightarrow \ell^{+} \ell^{-}$ decay into account, where $\ell^{+} \ell^{-}(2 \ell)$ stands for $\mu^{+} \mu^{-}$and $e^{+} e^{-}$combined.

\section{Parameter scans and event analysis}

We scanned the NMSSM parameter space, given in table 1, to search for regions yielding $m_{A_{1}} \lesssim 150 \mathrm{GeV}$ and the mass of $H_{2}, m_{H_{2}}$, around $125 \mathrm{GeV}$. We used the publicly available package NMSSMTools-v4.2.1 [4] for computation of the SUSY mass spectrum and branching ratios (BR) of the Higgs bosons for each model point. In our scans we imposed the constraints from $b$-physics, based on [6], and from Dark Matter relic density measurement [7], as

- $\mathrm{BR}\left(\mathrm{B}_{\mathrm{s}} \rightarrow \mu^{+} \mu^{-}\right)=(3.2( \pm 10 \%$ theoetical error $) \pm 1.35) \times 10^{-9}$,

- $\mathrm{BR}\left(\mathrm{B}_{\mathrm{u}} \rightarrow \tau v\right)=(1.66 \pm 0.66 \pm 0.38) \times 10^{-4}$,

- $\mathrm{BR}\left(\overline{\mathrm{B}} \rightarrow \mathrm{X}_{\mathrm{s}} \gamma\right)=(3.43 \pm 0.22 \pm 0.21) \times 10^{-4}$,

- $\Omega_{\chi} h^{2}<0.131(0.119+10 \%$ theoetical error $)$.

Exclusion limits from the LEP and LHC Higgs boson searches were also tested against using the HiggsBounds-v4.1.3 [8] package. Finally, from NMSSMTools we obtained the signal rates of $H_{2}$, defined for a given decay channel $X$ as

$$
R_{X} \equiv \frac{\sigma\left(g g \rightarrow H_{2}\right) \times \mathrm{BR}\left(H_{i} \rightarrow X\right)}{\sigma\left(g g \rightarrow h_{\mathrm{SM}}\right) \times \mathrm{BR}\left(h_{\mathrm{SM}} \rightarrow X\right)},
$$

where $h_{\mathrm{SM}}$ is the SM Higgs boson with the same mass as $H_{2}$. We then required $R_{X}$ for $X=\gamma \gamma, Z Z$ to lie within the measured $\pm 1 \sigma$ ranges of the corresponding experimental quantities $\mu_{X}$ by the CMS collaboration [5]. These ranges read

$$
\mu_{\gamma \gamma}=1.13 \pm 0.24 \text { and } \mu_{Z Z}=1.0 \pm 0.29 \text {. }
$$

Following the scans, we carried out a dedicated signal-to-background analysis based on Monte Carlo event generation for proton-proton collisions at $14 \mathrm{TeV}$ centre-of-mass energy at the LHC, for each process of interest. Using the program SuSHi-v1.1.1 [9], we first calculated the GF production cross section of an SM Higgs boson with the same mass as as that of a $H_{i}$ which is expected to decay 
into $A_{1} A_{1}$ or $A_{1} Z$ for a given SUSY point. This cross section was then rescaled using the $g g H_{i}$ reduced coupling in the NMSSM, and multiplied by the relevant BRs of the $H_{i}$, all of which are obtained from NMSSMTools. The backgrounds, which include the $p p \rightarrow 4 b, p p \rightarrow 2 b 2 \tau, p p \rightarrow 4 \tau$, $p p \rightarrow Z 2 b$ and $p p \rightarrow Z 2 \tau$ processes, were computed with MadGraph 5 [12]. Both the signal and the background for each process were hadronised and fragmented using Pythia 8.180 [10] interfaced with FastJet-v3.0.6 [11] for jet clustering. The parton-level acceptance cuts used are

- $|\eta|<2.5$ for all final state objects,

- $p_{T}>15 \mathrm{GeV}$ far all final state objects,

- $\Delta R \equiv \sqrt{(\Delta \eta)^{2}+(\Delta \phi)^{2}}>0.2$ for all $b$-quark pairs,

- $\Delta R>0.4$ for all other pairs of final state objects,

where $p_{T}, \eta, \phi$ are the transverse momentum, pseudorapidity and azimuthal angle, respectively.

Our use of the jet substructure method [13] implied that we had three possible signatures for a decaying $A_{1}$ : one fat jet, two single $b$-jets and two $\tau$-jets. The fat jet analysis, which assumes boosted $b$-quarks, allows one to obtain much higher sensitivities, particularly for large masses of the decaying Higgs bosons. We then calculated the expected cross sections for the signal processes which yield $S / \sqrt{B}>5$ for three benchmark accumulated luminosities at the LHC, $\mathscr{L}=30 / \mathrm{fb}, 300 / \mathrm{fb}$ and $3000 / \mathrm{fb}$, in various final state combinations, as functions of $m_{A_{1}}$.

\section{Results}

For the figures shown in this section, we first make two assertions: 1) all the points shown satisfy the constraints mentioned earlier and yield $122 \mathrm{GeV}<m_{H_{2}}<129 \mathrm{GeV}$, and 2) the sensitivity curve(s) shown corresponds to the best final state combination(s) for probing the given process.

Production via $H_{2} \rightarrow A_{1} A_{1} / Z$ : We begin with the decays of the SM-like $H_{2}$, since reconstructing its correct mass improves the kinematics, as noted earlier. In figure 1(a) we show the prospects for the $H_{2} \rightarrow A_{1} A_{1}$ channel at the LHC. Also shown are the sensitivity curves for the $2 b 2 \tau$ final state at $\mathscr{L}=30 / \mathrm{fb}$ and for the $4 \tau$ final state at $\mathscr{L}=3000 / \mathrm{fb}$. We see that a large part of the NMSSM parameter space can be probed via $H_{2} \rightarrow A_{1} A_{1}$ decays at the LHC, at $\mathscr{L}$ as low as $30 / \mathrm{fb}$. Note that the Higgs boson signal rate constraints from CMS restrict the $\mathrm{BR}\left(H_{2} \rightarrow A_{1} A_{1}\right)$ to less than $50 \%$. In figure 1(b) we see that the $H_{2} \rightarrow A_{1} Z$ decay shows no promise even at $\mathscr{L}=3000 / \mathrm{fb}$.

Production via $H_{1,3} \rightarrow A_{1} A_{1} / Z$ : The case of the $H_{1} \rightarrow A_{1} A_{1}$ decay, for a singlet-like $H_{1}$, is illustrated in figure 2(a). One sees that almost all the points with $m_{A} \gtrsim 12 \mathrm{GeV}$ are potentially discoverable in the $2 b 2 \tau$ final state at $\mathscr{L}=30 / \mathrm{fb}$. Two separate sensitivity curves corresponding to this final state indicate that for low $A_{1}$ masses the fat jet analysis has been employed, which results in a better reach. Even lighter $A_{1}$ could also be visible in the $4 \tau$ final state with $\mathscr{L}=300 / \mathrm{fb}$. Figure 2(b) shows poor prospects for the discovery of $A_{1}$ via the $H_{1} \rightarrow A_{1} Z$ channel also.

In figure 3(a) we see that the $H_{3} \rightarrow A_{1} A_{1}$ channel will be inaccessible at the LHC due to the fact that for such high masses of $H_{3}(\gtrsim 400 \mathrm{GeV})$ the production cross section gets diminished. 


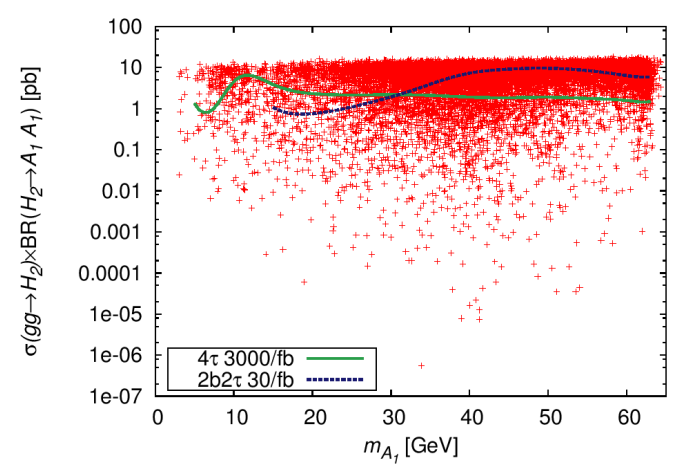

(a)

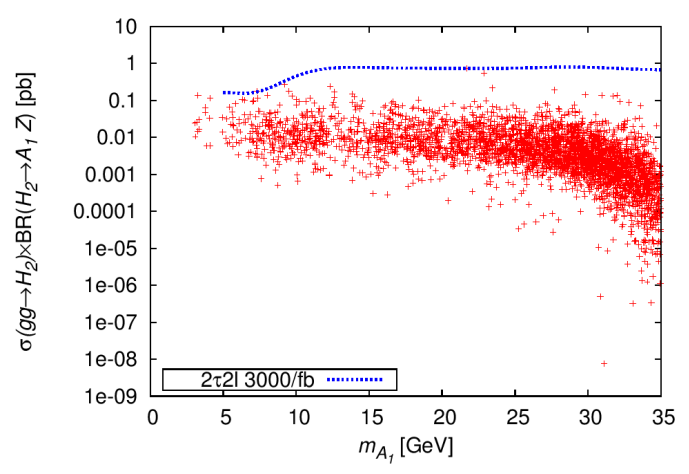

(b)

Figure 1: Cross sections for (a) the $g g \rightarrow H_{2} \rightarrow A_{1} A_{1}$ process and (b) the $g g \rightarrow H_{2} \rightarrow A_{1} Z$ process.

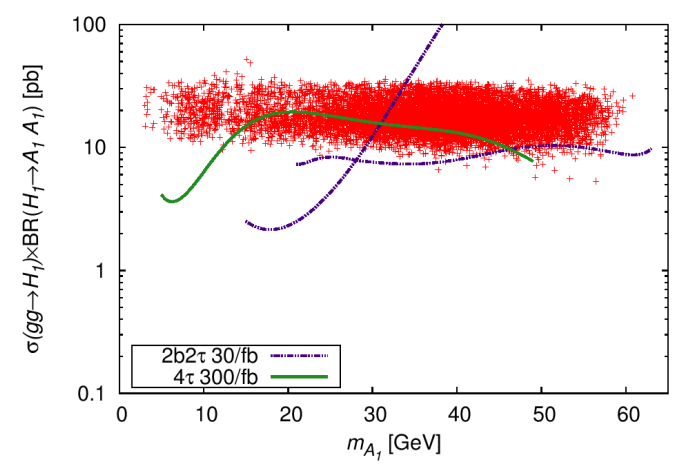

(a)

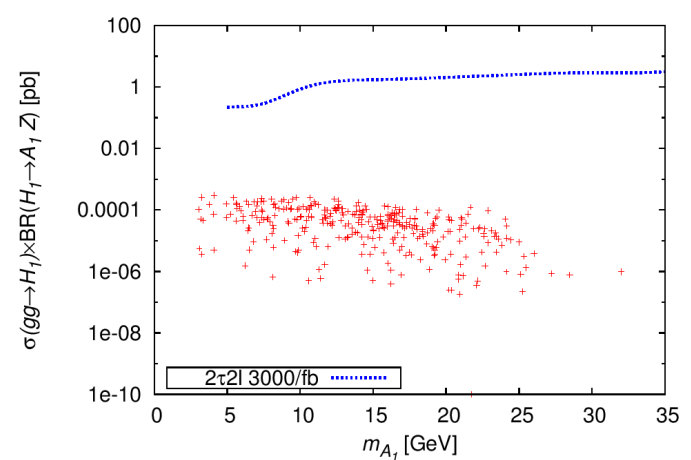

(b)

Figure 2: Cross sections for (a) the $g g \rightarrow H_{1} \rightarrow A_{1} A_{1}$ process and (b) the $g g \rightarrow H_{1} \rightarrow A_{1} Z$ process.

Moreover, other decay channels of $H_{3}$ dominate over this channel. The sensitivity curve in the figure corresponds to the $2 b 2 \tau$ final state for $\mathscr{L}=3000 / \mathrm{fb}$. Conversely, as shown in figure 3(b), in the $H_{3} \rightarrow A_{1} Z$ channel a number of points lie above the $2 b 2 \ell$ sensitivity curve for $\mathscr{L}=300 / \mathrm{fb}$. The discoverability of an $A_{1}$ in this channel results from the use of the fat jet analysis as well as from a sizeable $H_{3} A_{1} Z$ coupling, owing to a significant doublet component in $A_{1}$.

In summary, the decays of the two lightest scalar Higgs bosons carry the potential to reveal an $A_{1}$ with mass $\lesssim 60 \mathrm{GeV}$ for an integrated luminosity of $30 / \mathrm{fb}$ at the LHC. When the $A_{1}$ is heavier than $\sim 60 \mathrm{GeV}$, while its pair production also becomes inaccessible, the $g g \rightarrow H_{3} \rightarrow A_{1} Z$ channel takes over as the most promising one. This channel is, therefore, of great importance and warrants dedicated probes in future analyses at the LHC.

\section{Acknowledgments}

This work has been funded in part by the Welcome Programme of the Foundation for Polish Science. S. Moretti is supported in part through the NExT Institute. S. Munir is supported in part by the Swedish Research Council under contracts 2007-4071 and 621-2011-5107. The use of the CIS computer cluster at NCBJ is gratefully acknowledged. 


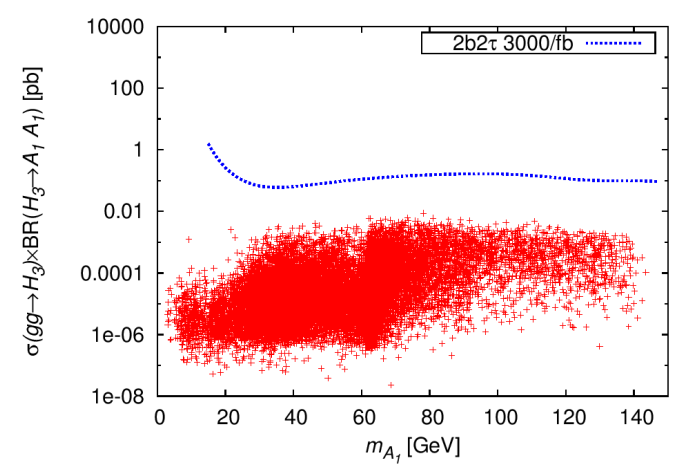

(a)

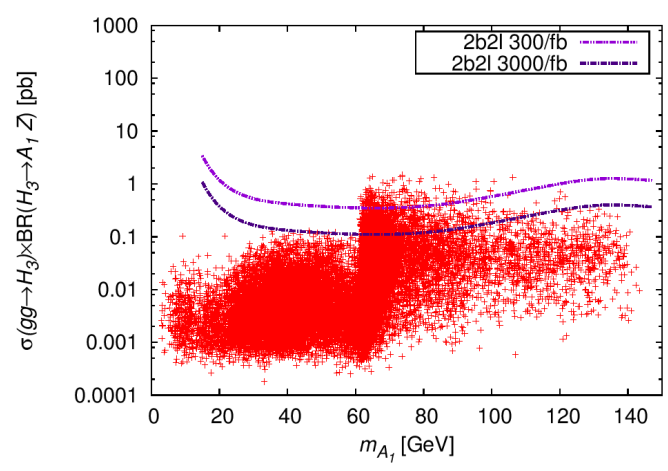

(b)

Figure 3: Cross sections for (a) the $g g \rightarrow H_{3} \rightarrow A_{1} A_{1}$ process and (b) the $g g \rightarrow H_{3} \rightarrow A_{1} Z$ process.

\section{References}

[1] S. Munir, L. Roszkowski and S. Trojanowski, Simultaneous enhancement in $\gamma \gamma, b \bar{b}$ and $\tau^{+} \tau^{-}$rates in the NMSSM with nearly degenerate scalar and pseudoscalar Higgs bosons, Phys. Rev. D88 (2013) 055017 [arXiv: 1305.0591$]$.

[2] S. Chatrchyan et al. (CMS Collaboration), Observation of a new boson at a mass of $125 \mathrm{GeV}$ with the CMS experiment at the LHC, Phys. Lett. B716 (2012) 30-61 [arXiv: 1207 . 7235].

[3] G. Aad et al. (ATLAS Collaboration), Observation of a new particle in the search for the Standard Model Higgs boson with the ATLAS detector at the LHC, Phys. Lett. B716 (2012) 1-29 [arXiv:1207.7214].

[4] www.th.u-psud.fr/NMHDECAY/nmssmtools.html.

[5] CMS Collaboration, Precise determination of the mass of the Higgs boson and studies of the compatibility of its couplings with the standard model, CMS PAS HIG-14-009 (2014).

[6] J. Beringer et al. (PDG Collaboration), Review of Particle Physics, Phys. Rev. D86 (2012) 010001.

[7] P. A. R. Ade et al. (Planck Collaboration), Planck 2013 results. XVI. Cosmological parameters, Astron. Astrophys. 571 (2014) A16 [arXiv:1303.5076].

[8] P. Bechtle et al., HiggsBounds-4: Improved Tests of Extended Higgs Sectors against Exclusion Bounds from LEP, the Tevatron and the LHC, Eur. Phys. J. C74 (2014) 2693 [arXiv:1311. 00 55].

[9] R. V. Harlander, S. Liebler and H. Mantler, SusHi: A program for the calculation of Higgs production in gluon fusion and bottom-quark annihilation in the Standard Model and the MSSM, Comput. Phys. Commun. 184 (2013) 1605-1617 [arXiv: 1212 . 3249].

[10] T. Sjostrand, S. Mrenna and P. Z. Skands, A Brief Introduction to PYTHIA 8.1, Comput. Phys. Commun. 178 (2008) 852-867 [arXiv: 0710.3820].

[11] M. Cacciari, G. P. Salam and G. Soyez, FastJet User Manual, Eur. Phys. J. C72 (2012) 1896 [arXiv:1111.6097].

[12] J. Alwall et al., MadGraph 5: Going Beyond,JHEP 1106 (2011) 128 [arXiv: 1106.0522 ].

[13] J. M. Butterworth, A. R. Davison, M. Rubin and G. P. Salam, Jet substructure as a new Higgs search channel at the LHC, Phys. Rev. Lett. 100 (2008) 242001 [arXiv: 0802 .2470]. 